\title{
LA TRADICIÓN MÍSTICA EN SAMPAIO BRUNO
}

\author{
MANUEL LÁZARO PULIDO \\ U. Francisco de Vitoria, España / U. Bernardo O’Higgins, Chile*
}

\begin{abstract}
RESUMEN: Sampaio Bruno (1857-1915) es uno de los autores culturales más significativos de Oporto y de todo Portugal. Su pensamiento filosófico-religioso se inscribe en el marco de un romanticismo tardío que se expresa de forma especial en su concepción mística. La mística de Sampaio Bruno expresa su romanticismo en la lectura ecléctica, antimetafísica y gnóstica de la tradición filosófica, que sin embargo confirma la riqueza de esa tradición en la búsqueda del infinito.
\end{abstract}

PALABRAS CLAVE: Sampaio Bruno; Renascença Portuguesa; mística; filosofía de la religión.

\section{Mystical tradition in Sampaio Bruno}

ABSTRACT: Sampaio Bruno (1857-1915) in one of the most important cultural authors in Porto and the rest of Portugal. His philosophical and religious thought, especially his mystical conception, can be inscribed in the last romanticism. The Sampaio Bruno's mysticism expresses the romanticism in an eclectic, antimetaphysical, and gnostic reading of the philosophical tradition. This reading, moreover, affirms the richness of the tradition itself in the infinity search.

KEY WORDS: Sampaio Bruno;Renascença Portuguesa; Mystic; Philosophy of religion.

\section{Introducción. Sampaio Bruno y la filosofía mística}

Hablar del pensamiento místico — de su presencia o de su ausencia en Sampio Bruno- nos remite a las consideraciones y reflexiones del profesor Afonso Rocha, especialmente al trabajo presentado para la consecución de su grado de licenciatura canónica en teología sistemática, presentada en 1996 y titulada $O$ mal e o misticismo idealista em Sampaio Bruno ${ }^{1}$. Este es el primer trabajo de una serie de reflexiones y de agudas consideraciones y (grandes) desarrollos realizados tras la tesis doctoral y que pueden verse en las obras $O$ mal no pensamento de Sampaio Bruno ${ }^{2}$, Natureza, razão

\footnotetext{
* El presente estudio tiene como base la comunicación «A Mística em Samapio Bruno», presentada en el Congresso «A Obra e o Pensamento de Sampaio Bruno». Universidade do Porto, Universidade Católica Portuguesa e Instituto de Filosofia Luso-Brasileira. Lisboa-Porto (Portugal), 4-6 de noviembre de 2015, cuyas actas se publicaron en 2018. El presente trabajo se inscribe dentro del proyecto de investigación financiado por el Departamento de Ciencias del Derecho de la Universidad Bernardo O'Higgins en consonancia con el Proyecto «Auditoría de las humanidades digitales: el desarrollo de su marco conceptual para la aplicación en proyectos académicos de investigación e innovación docente en los grados de la Facultad de Comunicación» de la Universidad Francisco de Vitoria.

1 Rосна, A., O mal e o misticismo idealista em Sampaio Bruno, dissertação apresentada a Faculdade de Teologia da Universidade Católica Portuguesa para a obtenção do grau de Mestre em Teologia Sistemática. Orientador: Prof. Dr. Arnaldo Cardoso de Pinho. Porto, 1996.

2 Rocha, A., O mal no pensamento de Sampaio Bruno, 2 vols., Imprensa Nacional - Casa da Moeda, Lisboa 2006.
} 
e mistério. Para uma leitura de Sampaio (Bruno ${ }^{3}$ y el capítulo VI de la obra A filosofia da religião em Portugal (1850-1910), titulada "A religião como "misticismo idealista": uma religião racional e de redenção»". Tras este ingente y lúcido trabajo parece que poca cosa se podría decir aquí. No obstante, cada persona tiene su forma de mirar, su perspectiva, y estas diversas formas de mirar dicen matices que a veces, solo a veces, pueden ayudarnos y pueden contribuir a un pensamiento bien realizado. Por este motivo hablamos de cuestiones en torno a la mística, y especialmente a la tradición mística.

Aunque Sampaio Bruno es un autor que no es nada ajeno a los estudios de historia de la filosofía portuguesa, quizás no es tan conocido para al resto de lectores y estudiosos peninsulares. Por lo que vamos a dedicar unas breves líneas biográficas 5 . José María de Sampaio (más tarde adoptó el seudónimo de Bruno) está ligado a la ciudad de Oporto (Porto) donde nació y murió (1857-1915), estudió - asistió al curso preparatorio de la Escuela Médico-Quirúrgica de la academia politécnica de Oporto (1876-1880) — y ejerció una vida polifacética: periodista su ocupación profesional en la que pudo desarrollar el resto de inquietudes, en los periódicos: A Folha Nova, A Discussão, A República, A República Portuguesa, A Pátria y Diário da Tarde—, polemista, crítico, filósofo y, diremos también, místico, ambas actividades, estas últimas, que ocuparán la segunda parte de su vida. Tuvo que abandonar su ciudad natal, exiliado por otra de sus actividades fundantes, como dirigente del movimiento republicano (1891), al que pertenecía desde la edad de 21 años y al que dedicó, horas y obras.

Su pensamiento intenta superar toda reminiscencia del «jesuitismo portugués», por expresarlo en las palabras que nacen a partir de la consecuencia de la reforma emprendida por el Marqués de Pombal, entendido como pensamiento escolástico en su sentido más tradicionalista, es decir, en tanto que pensamiento tradicional anclado en una metodología dominada por el argumento de autoridad, la elaboración desde el comentario y la transmisión del conocimiento nacida de la repetición. Esto supone que en el entorno del pensamiento portugués en el que se desenvuelve Sampaio Bruno se hacen presentes las corrientes filosóficas que en la época hacen furor y que van desde el positivismo de Comte, Littré o Spencer al neokantismo, al eclecticismo, al espiritualismo, al materialismo y al krausismo. Efectivamente en el Portugal del siglo XIX se establecerá un debate abierto entre cientificismo y metafísica a nivel teórico, debate que se extiende al pensamiento práctico que provoca la situación política y que exige un posicionamiento sobre el hombre y su posición social y política ${ }^{6}$. En ese ir y venir de ideas, Sampaio Bruno, junto a

\footnotetext{
3 Rocha, A., Natureza, razão e mistério. Para uma leitura de Sampaio (Bruno), Imprensa Nacional - Casa da Moeda, Lisboa 2009.

4 Rocha, A., A filosofia da religião em Portugal (1850-1910), Universidade Católica Editora Porto, Porto, 2013, pp. 391-567.

5 Un perfil del autor, su obra y pensamiento, lo tenemos en Serrão, J., Sampaio Bruno. O homem e o pensamento, Editorial Inquérito, Lisboa 1958.

6 Para este período tenemos la obra recopilatoria Calafate, P. (Dir.), Pimentel, M. C. (Coord.), Historia do Pensamento Filosófico. Volume IV: O Século XIX. 2 Tomos, Ed. Caminho, Lisboa 2004.
} 
Pedro de Amorin Viana ${ }^{7}$ y Domingos Tarrozo ${ }^{8}$, se presentan como espíritus libres que se oponen a toda filosofía de Escuela, no solo a la escolástica y al tomismo, sino también al positivismo francés y al idealismo alemán. De entre sus obras podemos citar Analyse da Crença Christã (Estudos Críticos sobre o Christianismo) $(1874)^{9}$, A Ideia de Deus $(1885)^{10}$, A Geração Nova (1886) ${ }^{11}$, O Brazil Mental (1898) $)^{12}$, O Encoberto (1904) $)^{13}$, Os Modernos Publicistas Portuguezes (1906) ${ }^{14}$ y A Questão Religiosa $(1907)^{15}$. Escritos que condensan en cierta forma las ideas que fue dejando en sus numerosos artículos como periodista, crítico y polemista. A pesar de su multifacético interés vital e intelectual, se podría definir el tenor existencial de Sampaio Bruno, desde su pulso intelectual, como hombre de pensamiento que se refleja de forma significativa en su filosofía mística ${ }^{16}$. Como se señalaba, al respecto, en el número especial dedicado al pensamiento portugués del Boletín Informativo de la Fundación C. Gulbenkian:

Em A Ideia de Deus, Bruno subordina a indagação filosófica a uma teologia heterodoxa, que explica o mal por uma misteriosa queda em Deus e que atribui ao homem a missão de regenerar essa queda, isto é, de ajudar a evolução da natureza. Mas o homem está ainda, de si próprio, encoberto. "O Encoberto e o Homem», diz. Descobrimento do homem nas suas virtualidades profundas, investimento do povo português e do homem português em tal missão transcendente e profética, enfim, uma nova religião da libertação universal. simultaneamente supra-humanizante e divinizante, eis os principais estádios da filosofia complexa de Sampaio Bruno $^{17}$.

En este sentido, traemos a la memoria las palabras del citado profesor Rocha al afirmar que:

Segundo Sampaio Bruno, os Cavaleiros do Amor são a expressão e o prolongamento de uma linha de pensamento místico-esotérico que, vindo de muito longe, da gnose antiga e judaica, através dos Templários, passou pela Cabala, pelos Élus-Cöhens, pelo Rosacrucianismo, pela Maçonaria, pela Revolução Francesa. Anunciando a Religião da Razão, o seu ideal e a sua luta estabelecem uma linha de

7 Amorim Viana, P., Defesa do Racionalismo ou análise da Fe, pref. A. Braz, text. A. C. Leal da Silva, Imprensa Nacional - Casa da Moeda, Lisboa 1982; Id. Escritos Filosóficos, ed. A. C. Leal da Silva, Imprensa Nacional - Casa da Moeda, Lisboa 1993. Cf. Soveral, E. A. de, "A situação de Amorim Viana (1822-1901) na História da Filosofia Portuguesa», en: Revista da Faculdade de Letras do Porto - Filosofia, $2^{\text {a }}$ série, 7 (1990), pp. 287-305.

8 Domingos Tarrozo, Filosofia da Existência. Esboço Sintético duma Filosofia Nova, Biblioteca do Notte Editora, Ponte do Lima 1881; Id., A Poesia Filosófica. Poemas Modernos, Biblioteca do Norte Editora, Ponte do Lima 1883. Cf. SÁ Do Rio, M. A., «Domingos Tarrozo (1860-1933) - Itinerário Filosófico», en: Revista Portuguesa de Filosofia, 34 (1978) pp. 339-358

9 Typ. De Arthur José de Sousa, Porto.

10 Livraria Chardron; Lello \& Irmão Editores, Porto.

11 Magalhães \& Moniz Editores, Porto.

12 Livraria Chardron, Porto.

13 Livraria Moreira Editora, Porto.

14 Livraria Chardron, Porto.

15 Livraria Chardron, Porto.

16 Rocha, A., A filosofia da religião em Portugal, o. c., p. 393.

17 «Os pensadores livres», en: Processo e Situação do Pensamento Português. Boletim Informativo Fundação Calouste Gulbenkian, Série II, 28 (1974), p., 289. 
continuidade e de afinidade com o catarismo e a Inquisição (medieval e moderna), como o Preste - João e com a Revolução Francesa ${ }^{18}$.

Estas referencias a las fuentes de Sampaio Bruno, así como a la superación de la Religión de la Razón de Kant, tras la influencia del misticismo del metafísico y místico Amorim Viana, expresan el tratamiento especial que Sampaio Bruno hace de la mística y el misticismo, lo que provoca, como señala Afonso Rocha que:

Diversamente, ao abandonar o racionalismo irreligioso, Bruno fará questão, quer de assumir expressa e frontalmente como um crente que não é católico, nem cristão, quer de fazer supor que uma tal posição tem a ver como razões de natureza histórico-fenomenológica e de natureza metafísico-teológica.

Em termos essenciais, Sampaio (Bruno) contraporá à religião cristã a conceição místico-gnóstica da religião, e isto, quer porque subsume o misticismo "gnóstico» como sendo a forma de religião que mais convém e que mais se adequa à humanidade, quer porque considera que certos fenómenos históricos do cristianismo, designadamente os relacionados com a intolerância e a Inquisição, não são compatíveis com uma religião que se reclama de ser a religião verdadeira, quer porque considera que algumas verdades e dogmas essenciais do cristianismo enfermam claramente de falta de racionalidade filosófica e teológica, quer porque sabe que a subsunção "gnóstica» da religião constituiu uma constância ao longo da história da humanidade ${ }^{19}$.

Por otra parte, la monografía de mitad del siglo XX, escrita por Joel Serrão sobre Sampaio Bruno, hacía referencia en el capítulo dedicado a los «Fundamentos gnoseológicos» a algunas de las características de su pensar:

o pensamento de Bruno tem raízes esotéricas, filia-se numa tradição acroamática que, a par e passo da filosofia moderna, racionalista e crítica, sub-reptìciamente continuou o ensino da cabala judaica, mística e emanancionista ${ }^{20}$.

Ambos textos hablan de la referencia tradicional, por así decir, del misticismo gnóstico de Sampaio Bruno. La reflexión que proponemos sobre la mítica de Sampaio Bruno, realizada a partir de la historia del pensamiento filosóficoteológico, intenta subrayar el carácter tradicional en el pensamiento de su tiempo, y enraizar su posición filosófica, en una de las tradiciones que recorre y atraviesa tangencialmente la historia del pensamiento filosófico y teológico como es el caso del pensamiento hermético.

\section{LA TRADICIÓN HERMÉTICO-MÍSTICA DE LA FILOSOFÍA ROMÁNTICA}

La lectura racionalista de la religión es una de las formas de abordaje del fenómeno religioso ${ }^{21}$. Se trata de realizar una interpretación religiosa a partir de las categorías de la razón. El hombre constituye, así, la base y el criterio último de

18 Rocha, A., O mal e o misticismo idealista, o. c., p. 303.

19 Rocha, A., A filosofia da religião em Portugal, o. c., pp. 415-416.

20 Serrão, J., Sampaio Bruno, o. c., p. 183.

21 Cf. Alston, W. P.; Romerales, E. (eds.), Creencia y racionalidade. Lecturas de filosofía de la religión, Anthropos - Consejo Superior de Investigaciones Científicas, Barcelona 1992. 
la legitimidad de la religión. Teniendo como origen las primeras diferenciaciones griegas frente a la mitología, en la modernidad el racionalismo religioso - y sus derivaciones afectivas-, está presente tanto en el idealismo, como en el romanticismo, influido por el pensamiento ilustrado. En este recorrido se pueden citar en el periodo antiguo el neoplatonismo y la gnosis. El primero -afirma Adriano Alessi- constituye «la religión, más filosófica, intelectual y aristocrática, en contraposición al carácter mítico y popular de la religión cristiana». Las sectas gnósticas, «continúan la pretensión neoplatónica de ofrecer una salvación esperable mediante el puro conocimiento ${ }^{22}$. A veces las fronteras de los conceptos no quedan claramente definidas. Estudiando las relaciones existentes entre hermetismo y simbolismo en la literatura romántica del siglo XIX, Massaud Moisés, subraya cómo el término hermetismo se mueve en un gran campo semántico «poblada por una serie de conceptos próximos, incluso a riesgo de ser tomados por equivalentes», sería el caso de términos y conceptos como esoterismo, ocultismo, neoplatonismo, misticismo, gnosticismo, alquimia, cábala, magia ${ }^{23}$. Ciertamente, existe una confusión intrínseca a la propia utilización de los diferentes autores en las diversas épocas del pensamiento y en sus distintas disciplinas, bien sea la filosofía, bien la literatura, bien el pensamiento religioso, bien en la convergencia de todas ellas.

La lectura de las obras de Sampiao Bruno tiene para no pocos lectores este sabor de filosofía, religión, ensayo literario y todo en uno, propio de un pensamiento cercano al hermetismo y que no es ajeno al sentir del contexto en el que se desarrolla su pensamiento y sus lecturas. Lo que podría interpretarse como ideas «confusísimas e incoherentes, pero llenas de chispas y de vislumbres», al decir de Unamuno, sin embargo adquieren más sentido si volvemos la mirada a los autores del siglo XIX. La deuda intelectual de Sampaio Bruno con Friedrich Schelling a través de Karl Robert Eduard von Hartmann — que Amorim de Carvalho señala en su reflexión sobre el positivismo metafísico de Sampaio Bruno, hablando de la influencia "poderosa» del metafísico alemán Eduardo de Hartmann²4 - y su referencialidad, no es casual. Manuel Gama se refiere a esta vinculación:

Pelas indicações que nos dá, o seu esquema de cariz místico ter-se-á inspirado nas ideias de E. de Hartmann, expostas na sua obra Filosofia do Inconsciente, que por sua vez faz eco do pensamento do filósofo alemão Schelling, que sintetiza determinadas tendências da cultura ocidental. Tendências essas, de cariz neoplatónico, próximas dos sistemas gnósticos heterodoxos, e veiculadas por Jacob Boehme, um dos expoentes da mística especulativa alemã dos séculos XVI-XVII, que procura conciliar o absoluto com a existência da realidade finita e respectiva presença do $\mathrm{mal}^{25}$.

22 Alessi, A., Los caminos de lo sagrado. Introducción a la filosofía de la religión, Ed. Cristiandad, Madrid 2004, p. 169.

23 Moisés, M., «Hermetismo y simbolismo. Aproximaciones», en: Beltrán, L.; RodríGuez, J. L. (coords.), Simbolismo y hermetismo. Aproximación a la modernidad estética, Prensas Universitarias de Zaragoza, Zaragoza 2007, p. 14.

24 Cf. Carvalho, A. de, O Positivismo metafísico de Sampaio Bruno, Fundação Lusíada, Lisboa 2001 .

25 Gama, M., "Sampaio Bruno - Heterodoxia e humanismo», o. c., p. 4. Texto de la comunicación presentada en el Coloquio «O Pensamento Filosófico Português no Século XIX», en el ámbito de la presentación del vol. IV de la História do Pensamento Filosófico Português, que se celebró en 
No extraña, así, el apelativo de «Schelling portugués», utilizado por Vemireh Chacon $^{26}$. Estas referencias intelectuales no afectan solo al concepto de naturaleza, sino que también penetran su «mente» $\mathrm{y}$ «mentalidad» cuando permanece delante del universo mental, el que se desarrolla cuando siendo prácticamente un adolescente siente las ganas de escribir bajo el pseudónimo de Bruno haciendo referencia a Giordano Bruno, aquel que sufrió, recuerda, «el suplicio» ${ }^{27}$, aquel que encarna el anticlericalismo romántico de los versos de Guilherme de Braga, determinantes a la hora de escoger el nombre con el que firmar los artículos ${ }^{28}$. Y lo que nace como una referencia que expresa la rebeldía contra el orden (el del catolicismo y el jesuitismo - y como expresión del Padre que era «hombre de orden e de método» ${ }^{29}($ ?) - ) , se vuelve a su vez referencia de pensamiento, lo que cobra sentido en su tiempo.

Cuando hablamos de tradición mística en Sampaio Bruno no nos quedamos única y exclusivamente referenciados al imaginario místico-tradicional en la Península nacido de la experiencia vivida, escrita, y muy leída por los místicos «cristianos» del Siglo de Oro: Fray Luis de Granada, Teresa de Ávila o Juan de la Cruz. El misticismo presente en el siglo XIX mira y tiene en vista de forma muy especial a otro misticismo. Este misticismo que podemos llamar «romántico» posee otras fuentes. A veces también tiene presente el misticismo del recogimiento y carmelitano, ya que, en cierta forma, esta mística usa la inspiración común de naturaleza neoplatónica ${ }^{30}$ y se enfrenta - presentándose como una alternativaa la forma de pensar de la metafísica escolástica ${ }^{31}$. A veces este misticismo —que podemos chamar "teosófico»— hace una lectura «hermética» de los clásicos místicos cristianos.

El misticismo teosófico está presente en el idealismo de tendencia romántica y en el propio romanticismo. Schelling es el clásico ejemplo de esta tendencia, su paso por el Seminario de Tubinga, más allá de proporcionarle excelentes condiscípulos —Hölderin y Hegel—, le introduce en el mundo de la tradición teosófico-mística fundamentada en el pensamiento del Renacimiento. A partir de su obra Investigaciones Filosóficas sobre la Esencia de la Libertad Humana acrecienta la tendencia al misticismo. Permeabiliza su pensamiento con fuentes místicas como el misticismo romántico de Fichte, estableciendo una linea de misticismo que nace en el neoplatonismo y la gnosis griega hasta Jacob Böhme, a

la Faculdade de Letras da Universidade de Lisboa, el 7 y 8 de abril de 2005. Disponible en https:// repositorium.sdum.uminho.pt/bitstream/1822/28577/3/SB-(ABT)-txt\%20def..pdf, consultado el 23 de septiembre de 2017.

26 Chacon, V., «Sampaio Bruno: um Schelling português», en: Barreto, L. A. (org.), Colóquio Antero de Quental dedicado a Sampaio Bruno, Secretaria de Estado da Cultura de Sergipe, Aracaju 1995, pp. 275-288.

27 Bruno, S., «Carta íntima», en: A Idéia de Deus, o. c., p. XXXVI.

28 Ib., p. XXXVIII.

29 Ib., p. XXXVII.

30 Cf. LÁzAro, M., «Mística y filosofía. Silencio, escucha, palabra: El recogimiento franciscano», en: Cunha, J., Natário, M. C., Epifânio, R. (coords.), Palabra, Escuta e Silêncio. Filosofia, Teología e Literatura, Universidade Católica Editora, Porto 2014, pp. 249-260.

31 Cf. Lázaro, M., «Dos perspectivas del alma en el siglo XVI: los Conimbricensis y la mística de Fr. Juan de los Ángeles OFM», en: Anales del Seminario de Historia de la Filosofía, 31 (2014), pp. 347-372. 
través de los autores místicos renanos de la escolástica medieval y del pensamiento de Spinoza. El romanticismo se presenta como alternativa al empacho de razón ilustrada, pero a pesar de ello, es digno discípulo de los ilustrados en sus tendencias menos racionalistas. Los románticos beberán de la fuente que alimenta el resto de la vitalidad de la época de la Ilustración: la mística. Lejos de la idea - y casi el preconcepto - de una Ilustración definida por su carácter racional, liberal, teísta, antimetafísico, e incluso ateo, la verdad es que los ilustrados tendrán presentes en su pensamiento la vitalidad del misticismo:

Cábala, hermetismo y la llamada religiosidad emocional ofrecen técnicas para alcanzar la realidad espiritual más allá de la experiencia ordinaria. En esta dirección, la regeneración del hombre se busca en la experiencia mística, religiosa o simplemente espiritual, y ello da lugar a una producción escrita que constituye una «filosofía oculta», así llamada bien por su carácter heterodoxo frente a las ortodoxias religiosas existentes bien por su origen o parentesco hermético, o vinculación a una filosofía natural no mecanicista ${ }^{32}$.

La presencia de Jacob Böhme tiene que ser subrayada. Jacob Böhme representa la mística teosófica que marca parte de la modernidad postkantiana y romántica. El zapatero de Görlitz influyó en autores como Newton, Oetinger, Novalis, Schelling, Hegel, Goethe o Feuerbach en el siglo XIX siendo reconocido en el siglo XX por filósofos e intelectuales como Erns Bloch y Maria Zambrano. Más allá de inspirar el idealismo alemán y presentarse frente a una lectura material de la dialéctica, su pensamiento representa también un signo vital frente a la persecución. Los cristinos libres de los Países Bajos serán lectores de las obras del pensador alemán, pero también lo serán los pietistas e espiritualistas del siglo XVII y en Inglaterra los llamados «Bohemist Philadelphians» ${ }^{33}$. Su pensamiento religioso se define por la originalidad y la imaginación, a partir de la lectura de la mística renana. Aunque no siguiera un recorrido académico, había estudiado la Biblia, las obras de Paracelso ${ }^{34}$ y los tratados místicos, lo que hace que, junto a sus predecesores Valentin Weigel y Kaspar Schwenkfeld, sea inspirador de las lecturas espiritualistas y outsider del luteranismo alemán ${ }^{35}$. Su pensamiento no es ajeno a la lectura neoplatónica y cabalística del Paracelso. Jacob Böhme es sucesor de la tradición espiritual promovida por el Maestro Eckhart. Erns Bloch, nos recuerda Agustín Andreu Rodrigo, ve en su idealismo las ideas de Proclo y Dioniso-Areopagita. Señala además el introductor a la traducción de Aurora de M. Zabrano que la obra

32 CAnterla, C., «Neoplatonismo, filosofía natural y misticismo: fuentes ocultas del romanticismo en el Kant precrítico», en: Cuadernos de Ilustración y Romanticismo, 1 (1991), p. 163.

33 SchneIder, H., German Radical Pietism, trad. Gerald MacDonald, Scarecrow Press, Lanham 2007, pp. 6. Cf. Obst, H., «Jacob Böhme im Urteil Philipp Jacok Speener», en: Zeitschrift für Religions - Und Geistesgeschichte, 23 (1971), pp. 22-39.

34 GILly, C., "Das Bekenntnis zur Gnosis von Paracelsus bis auf die Schüler Jacob Bömnes», en: Broeck, R. van den, Heertum, C. van (eds.), From Poimandres to Jacob Böhme: Gnosis, Hermetism and the Christian Tradition, In the Pelikaan, Amsterdam, 2000, pp. 385-425.

35 Greyerz, K. Von, Religion and Culture in Early Modern Europe, 1500-1800, trad. Thomas Dunlap, Oxford University Press, New York 2007, p. 84; Van Horn Melton, J., «Pietism, Print Culture, and Salzburg Protestantism on the Eve of Expulsion», en: Strom, J., Lehmann, H., VAN Horn Melton, J. (eds.), Pietism in Germany and North America 1680-1820, Ashgate Publishing, Ltd., Fraham 2009, p. 244. 
se inscribe en la «tradición de Plotino y el Areopagita, el Zohar y Llull, de san Juan de la Cruz y de Molinos ${ }^{36}$. No debe extrañarnos la relación entre mística teosófica y neoplatonismo. Spinoza como autor de transición y Giordano Bruno como paso necesario hacia la modernidad marcarán el pensamiento de Jacob Böhme antes, y de los románticos después. La tríada misticismo-teosofía-neoplatonismo sigue una secuencia que conoce su origen en Giordano Bruno.

\section{Mística y metafísica en el Giordano Bruno de Sampaio Bruno}

El personaje de Giordano Bruno que fue elegido por el «Bruno» portugués por la representación que su muerte tiene como expresión de la resistencia al pensamiento imperante, es además inspiración del hermetismo romántico que aparece en el misticismo metafísico de nuestro Bruno. El hermetismo de Giordano Bruno fue subrayado por el célebre libro de Frances A. Yates, en la década de los 60 del siglo XX que realizó una lectura hermética del Renacimiento y, especialmente, de Giordano Bruno y la relación entre un tipo de misticismo mezclado con el gnosticismo en la antigüedad tardía y que, al decir de la autora, perdurará hasta el mismo Giordano Bruno y más allá ${ }^{7}$. El personaje de Giordano Bruno abarca una mitología personal, de la que sin duda bebió Sampaio Bruno. Nos encontramos ante un personaje que es, a la vez, objeto de la mitomanía, en torno a la magia, el hermetismo, la tradición gnóstica... Bruno, Giordano, es sin embargo, representante de una tradición antigua inspiradora de la modernidad: el poder del dominio del saber por parte del sujeto. En esta encrucijada podemos comprender por qué en Giordano Bruno existe una reductibilidad racional del hecho religioso, explicado a partir de una razón del conocimiento, donde la realidad tiene que ser profundizada desde una filosofía alejada de la magia, entendida en el sentido de magia transnatural ${ }^{38}$, que es propia y apropiada para el vulgo ${ }^{39}$. Porque la magia en Giordano Bruno es más polisémica. Se trata, así, de una razón capaz de gnosis y próxima al hermetismo. Se trata, por lo tanto, de una magia de la memoria, del orden:

Magia profunda — sustenta Carlos Muñoz sobre Giordano Bruno- es saber desgajar al contrario después de haber hallado el punto de unión.

El conocimiento como comprensión de la unidad, que es la naturaleza, posibilita la magia que es actuar como ésta, desgajando los contrarios... La memoria mágica bruniana pretende, entonces, poseer el orden, conexión, composición y gobernación de todas las cosas, de la relación entre lo superior y lo inferior; lo

36 Rodrigo, A. A. «Nota previa», en: Böhme, J., Aurora, Siruela, Madrid 2012, p. 13. La vinculación del misticismo hermético con el misticismo clássico viene muchas vesses del propio vocabulário: «El vocabulario con que traducir a Böhme al castellano de su siglo está todo él en Luis de Granada, en Cervantes y en Luis de la Puente». Ib., p. 14.

37 Yates, F. A., Giordano Bruno and the Hermetic Tradition, University of Chicago Press, Chicago 1964.

38 Cf. "Introduction on magic», en: Bruno, G., Cause, Principle and Unity, And Essays on magic, ed., trad., Robert de Lucca; Richard J. Blackwell, introd. Alfonso Ingegno, Cambridge University Press, Cambridge 1998, p. 106.

39 Bruno, G., De l'infinito universo e mondi, en Aquilecchia, G. (ed.), Dialoghi italiani. Dialoghi metafisici e dialoghi morali, G. C. Sansoni, Firenze 1958, pp. 386-387. 
material y lo espiritual y de su recíproca conversión que implica el ascenso y el descenso por la escala del $\operatorname{ser}^{40}$.

La lectura hermética del pensamiento de Giordano Bruno fue subrayada por la obra ya referida de Francis A. Yates. Su interpretación resulta interesante porque refleja una situación historiográfica y propia del criticismo literario común en la primera década del siglo XX muy interesada en la tradición Hermética y en la que resalta de forma significativa Giordano Bruno y su influencia en esta tradición desde el Renacimiento ${ }^{41}$. Pero reducir la lectura de Bruno al hermetismo no sería algo apropiado. Su lectura no ortodoxa del neoplatonismo y la extensión del alma del mundo y las estructuras vitales de la naturaleza, del cosmos y de Dios no impiden una metafísica filosófica ${ }^{42}$. Así la «magia profunda» de Bruno es de facto la lectura de los contrarios en un único infinito (Dios y naturaleza) en una interpretación que sigue las intuiciones del Maestro Eckhart y Nicolás de Cusa sobre la unidad de los contrarios del mundo, para resaltar la presencia de la eterna infinitud total divina en la inacabada infinitud total en la naturaleza. La resolución puede tener resonancias herméticas, pero el problema es metafísico, de la idea neoplatónica en un primer momento; racionalista después en las especulaciones de Spinoza. En esta dialéctica entre idealismo (neoplatónico) y misticismo (epistemológico) ${ }^{43}$ cabe hacer una lectura del pensamiento de Giordano Bruno y su múltiple influencia y divergencia de las lecturas.

Giovani Gentile, en el período más idealista, reconoce esta dualidad y manifiesta su lectura en su obra Giordano Bruno e il pensiero del Rinascimento, donde manifiesta

Il suo vero amore è l'amore dell'eterno e del divino l'amor Dei intellectualis, onde precorse quel grande mistico della filosofia intellettualistica, che fu nel secolo successivo Benedetto Spinoza. Nuovo misticismo, che male fa raccostare il nostro filosofo ai neoplatonici, benché sia innegabile, anzi notevolissimo, l'influsso della loro filosofia su quella del Bruno. La conoscenza del divino propugnata dal Bruno non è estasi, o unione immediata, benché abbia per suo termine appunto l'unione, onde lo spirito, egli dice, «doviene un dio dal contatto intellettuale di quel nume oggetto»); ma è un processo razionale, un discorso dell'intelletto una vera e propria filosofia ${ }^{44}$.

En esta dialéctica intervienen las ideas cabalísticas de Giordano Bruno, muy influidas por el interés de los humanistas del Renacimiento —especialmente el círculo Neoplatónico- por las culturas de Oriente. Así en el tiempo de Giordano Bruno, la Cabala es estudiada. «The Humanists — escribe De León-Jones— studied ancient texts from Egypt and Chaldaea, and of Jewish Kabbalah, including the

40 Muñoz, C., "Giordano Bruno: el Arte de la Memoria», en: A parte rei 12, p. 5. Disponible en serbal.pntic.mec.es/ cmunoz11/artebru.pdf, consultado o 23 de septiembre de 2017. Cf. YATES, F. A., The Art of Memory, Routledge; Kegan Paul, London 1966 (ed. en español: El arte de la memoria, trad. Ignacio López de Liaño, Ed. Siruela, Madrid 2005).

41 Cf. Jones, M. G., Frances Yates and the Hermetic Tradition, Ibis Press, Lake Worth, Fl. 2008.

42 Cf. Bartolomé, M., «El animismo en Giordano Bruno», en: Agora, 19 (2000), pp. 23-49.

43 Vессніотт, I., "Giordano Bruno fra idealismo e misticismo», en: Vecchiotti, I., Premessa a Jacobi seguita da un saggio sul Bruno, Corso, Roma 1959, pp.131-175.

44 Gentile, G., Giordano Bruno e il pensiero del Rinascimento, Valecchi Editore, Firenze 1925, p. 14 . 
famous Hermetica, which was erroneously dated to pre-Christian times, and the Zohar, which was thought to have been written by the patriarch Abraham» ${ }^{45}$. Tenemos un ejemplo en Pico de la Mirándola y su lectura de la Cabala cristiana como "disciplina mística». Chaim Wirszubski, en un estudio sobre el autor de Mirándola, permite comprender la confusión provocada en las lecturas posteriores de la cabala cristiana en la búsqueda de la conjunción de los elementos: «judaísmo e cristianismo, cristianismo e platonismo, mística e magia ${ }^{46}$. Las palabras de Walter Benjamin recordadas también por Chaim Wirszubski en referencia a Pico de la Mirándola sirven para comprender la metafísica neoplatónica y hermética en discusión en Giordano Bruno: «la unidad de tiempo de la experiencia simbólica y el instante místico ${ }^{47}$, aún más, ampliamos, en la unidad del espacio-tiempo, o sea, en la infinitud.

La confusión, y en ella la fascinación, continúa en el siglo XVII, entre los filósofos y teólogos, y se amplía en el siglo XIX. Anna Foa y Eugenio Canone entre otros han estudiado su influencia en la Italia del siglo XIX ${ }^{48}$. Los estudios recuperan la lectura mas filosófica de la influencia de Bruno sobre Schelling recuperando las consecuencias filosóficas del pensamiento de Bruno (y Spinoza). Así Schelling y Bhöme ven en las especulaciones filosófico-místicas de Bruno un apoyo para su filosofía de la identidad y una forma de poder formular una fundamentación del absoluto incondicionada que pudiese ir más allá de los límites del mecanismo regulador de la razón kantiana y explorar así el infinito y lo sublime ${ }^{49}$. Schelling rescata de este modo el Giordano Bruno filósofo.

Y este Bruno, Giordano, filósofo-místico en la idea de sabiduría, se presenta en su lectura de un Bruno quemado en la hoguera por la idea intrínseca de alternativa al poder eclesiástico-intelectual establecido. Lejos de las interpretaciones de algunos pensadores del siglo XIX, positivistas, que querían apropiarse de un esfuerzo científico cuando de hecho, nos recuerda Miguel Ángel Granada: «... ciertamente la condena no era una condena de la ciencia, sino de un hereje anticristiano» ${ }^{50}$.

El círculo se completa: el neoplatonismo, la gnosis, la mística y el pensamiento ibérico esta vez coinciden ${ }^{51}$ : en una metafísica del infinito, más allá de las constricciones ideológicas del concepto tanto escolástico-jesuítico, como científicopositivo. Unamuno y Sampio Bruno coinciden en esta idea, manifiesta José Manuel

45 De León-Jones, K. S., Giordano Bruno and the kabbalah: prophets, magicians and rabbits, Yale University Press, New Haven 1997, p. 7.

46 WirszuBsKI, Ch., Pico della Mirandola's encounter with Jewish mysticism, Harvard University Press 1989.

47 Benjamin, W., Ursprung des deutschen Traverspiels, Franckfurt an Main, 1963 p. 183, citado em Wirszubski, Ch., Pico della Mirandola's, o. c., p. 172.

48 FoA, A., Giordano Bruno, Il Muolino, Bologna 1998; Canone, E. (ed.), Bruno redivivus: momenti dela fortuna di Giordano Bruno nel XIX secolo, Edizioni dell'Ateneo, Pisa 1998.

49 JiménEz, J., «Del infinito al inefable. Tergiversación fenomenológica de la escritura bruniana», en: Granada, M. Á. (ed.), Cosmología, teología y religión en la obra y en el proceso de Giordano Bruno. Actas del congreso celebrado en Barcelona, 2-4 de diciembre de 1999, Edicions Universitat Barcelona, Barcelona 2001, p. 96.

50 Granada, M. Á., «Introducción», en: Giordano Bruno, La cena de las cenizas, trad., not. e introd. Id., Alianza, Madrid 1987, p. 9.

51 Cf. Sinnige, Th G., Neoplatonisme en Spaanse mystiek: Plotinos, Gnosis, Juan de la Cruz, Unamuno, Van Gorcum, Assen 1994. 
de Barros, más allá de la disputa sobre $O$ Encoberto. Ambos aprecian a Giordano Bruno por su espíritu combativo ${ }^{52}$ como «Quixote do pensamento». Pero ambos se alejan a la hora de interpretar el sentimiento del misticismo. Donde en Unamuno coinciden «humorismo» $\mathrm{y}$ "misticismo» ${ }^{53}$, en Sampaio Bruno el misticismo es acceso a la realidad del infinito.

Para terminar y a modo de conclusión, con Sampaio Bruno, recuperamos la tradición en su complejidad de lecturas, con el fin de comprender mejor, de forma más pausada, recordando lo escrito en el capítulo sobre el infinito perfecto en la obra a Idéa de Deus:

A conclusão geral de minha inquirição philosophica, pois, esta dupla: O Dualismo é falso, porque a Creação é um absurdo, porque do Nada não se póde dizer... O Monismo é falso, porque ou é irreal ou é irracional. O Monismo é atheista? É falso, porque o universo, só por só, desde que não ha Deus, é inteligível, vistocomo, resolvendo-se em sciencia, consoante effectivamente se resolve, mostra ser um systema de idéas, as quaes, no hypothese, emergiam então do nada. O Monismo é pantheista? E' falso, porque a immanencia substancial do Absoluto (ou deus) no universo não se coaduna com a evolução progressiva do mesmo universo, onde cada momento do progresso nega o anterior, por insuficiente, quando a substancialidade divina teria de ser, sob pena de contradicção no lemma basilar, a quietude da perfeição ${ }^{54}$.

Universidad Francisco de Vitoria, Madrid (España)

Universidad Bernardo O’Higgins, Santiago de Chile

manuel.lazaro@ufv.es

Manuel Lázaro Pulido

[Artículo aprobado para publicación en enero de 2019]

52 Dias, J. M., «Miguel de Unamuno y Sampaio Bruno: una polémica olvidada sobre O Encoberto", en: Flores, C. (coord.), Tu mano es mi destino. Congreso Internacional Miguel de Unamuno, Ediciones Universidad de Salamanca, Salamanca 2000, p, 131.

53 Bénédicte Vauthier recuerda una cita del epílogo de Amor y pedagogia: «Dejo a los místicos y a los humoristas —que son lo mismo-que escudriñen, pesquisen y requisen este simbolismo». Cita en Vauthier, B., Arte de escribir e ironía en la obra narrativa de Miguel de Unamuno, Ediciones Universidad de Salamanca, Salamanca 2004, p. 243.

54 Sampaio Bruno, A Idéia de Deus, o. c., pp. 398-399. 